When taken to hospital, he was found unable to move, stand upright, or otherwise maintain himself without assistance; the pupils were fixedly dilated, and almost insensible to light ; there was some ptosis of the left lid; the sphincters had lost their contractility; he moved as if in pain, started involuntarily, and "talked all idly unto shapes of air". $\mathrm{He}$, however, still retained some glimmering trace of consciousness, and could answer falteringly when spoken to in a loud voice, but soon relapsed into his original forgetfulness. His motions and general contour became jactitating and convulsed, his breathing more laboured and puffing; and, notwithstanding the use of a turpentine injection, the application of heat, friction, and stimulating poultices, and the exhibition of small and frequent doses of brandy, he had frequently recurring attacks of faintness and syncope, with cold perspiration, spasmodic twitchings, and pallor of face; and died convulsed soon afterwards.

Post Morten Examination, thirty-six hours after death. The body was moderately plump and muscular, and compara. tively free from cadaveric rigidity. There was no odour or trace of decomposition, and no perceptible discoloration or congestion about the face, neck, or scalp. On removing the latter, the cerebral membranes appeared bulging and congested; the tegumentary vessels were enlarged, tortuous, and superficially prominent from blood-engorgement; and there was a consider able amount of cerebro-spinal and subarachnoid fluid in the cavity of the skull. Both ventricles appeared unusually large, as if from fluid dilatation; there was a pinkish ruddiness of the cortical substauce, and numerous puncta vasculosa in the centrum ovale. Several small wedge-shaped tubercles were found dotting the surface of the arachnoid, giving it a distinct character of roughness and inequality, and being especially prominent towards the base of the brain and cerebellum. The encephalic mass was otherwise apparently quite normal. The left lung was rather freely studded with grey tubercle, and there was a small circumscribed vomica at its very apex. The bronchial, mesenteric, and cervical glands were enlarged, and infiltrated with softened curdy matter resembling broken down tubercular substance or scrofulous débris; and the abdominal organs, so far as could be ascertained, were generally free from active disease.

Remarks. On glancing cursorily over the reports of these two cases, it will be found that, as noticed by Dr. Hill, the symptoms hore no very distinctive or definite relationship; and one would be at some loss in accounting for their sequence, or even in approximatively estimating their value by a reference to or comparison of the phenomena of their development, progress, or termination. Though somewhat obscured by the in. fluence of protracted stimulation in the first case, they were otherwise certainly more characteristic and intensified than in the second, in which the outward appearances might be com. pared, on casual inspection, to those produced by or resulting from chronic encephalitis, cerebral abscess, or the presence of an entozoon. The pain in the head, the gastric irritation, the vomiting and prostration, the dead, dull, heavy, persistent sense of uneasiness and discomfort, as described by Dr. Brittan in connexion with a case of the last mentioned complication were quite compatible with the feelings of my second patient; while, in some other respects, his sufferings and genera appearance were not much at variance with those referred by Dr. Richardson to uræmia.

From these remarks, it will be easily inferred that the diagnosis of meningeal tuberculosis is during life to a certain extent uncertain and conjectural; and though, doubtless, per. sistent headache, with more or less paralysis and delirium, especially if the latter manifest itself by very wild and maniacal delusions, may, in a tuberculous patient, warrant a suspicion of its existence, and enable or justify us in taking measures accordingly, I yet fear that, in the present state of our knowledge, a physical examination alone will confirm or disprove the accuracy of our assumption. The presence of tubercular matter, and its recognition by the microscope, must even in death be our guide towards diagnosis; for, as will appear from the following words of Rokitansky - with which, as they entirely reflect my own ideas on the subject, I will close this paper-the presence of ventricular or subarachnoid fluid does not affect the question, or is rather obviously beside it, without the tests just referred to.

"It is an important question whether there are any acute effusions which are rapidly fatal, and whether it is possible to recognise them on the dead body as the cause, or as part of the cause, of that form of sudden death which is usually designated 'serous apoplexy'. That there are snch acute effusions, which may quickly destroy life by paralysing the brain, can oertainly. in the present state of our knowledge, not be denied; but the diagnosis of this mode of death is just as uncertain on the dead body as on the dying patient; for the serum which is found in the arachnoid sac may be a chronic accumulation which has existed there for a long time, just like that which is contained in the ventricles or in the tissues of the pia mater, or that which produces $\propto$ dema of the brain itself."

These observations warrant, I think, the conclusion just enunciated, and justify me in assigning the death of these two men to the irritation of tubercular matter in the meninges of the brain.

Pembroke Dock, South Wales, December 1860 .

OBSERVATIONS ON THE MORBID ANATOMY, PATHOLOGY, AND DETERMINING CAUSE OF EMPHYSEMA OF

\section{THE LUNGS.}

By A. T. H. WATERS, M.R.C.P., Physician to the Liverpool Northern Hospital ; Lecturer on Anatomy, Physiology, and Pathology, in the Liverpool Royal Infirmary School of Medicine.

[Read before the Royal Medical and Chirurgical Society.]

PART I.

The different kinds of Emphysema. Vesicular Emphysema; its various forms. Modes of Preparation of Lung Tissue for examination. Changes which take place in the Air-Sacs in the different Stages of the Disease. Condition of the Blood-Vessels; of the Bronchial Tubes. Is the Disease attended with any Degeneration of Tissue? Interlobular Emphysema.

[Continued from $p .959$.

THE subsequent steps in the progress of the disease consist in a further distension of the air-sacs, an enlargement of their perforations, and a rupture of the fibres of which their walls are composed. As these results take place, the walls become more and more imperfect, and the openings in them coalesce. A further breaking down of the walls then occurs, so as to leave but very partial partitions between the cavities; and in the most advanced stages of the disease these partitions un.

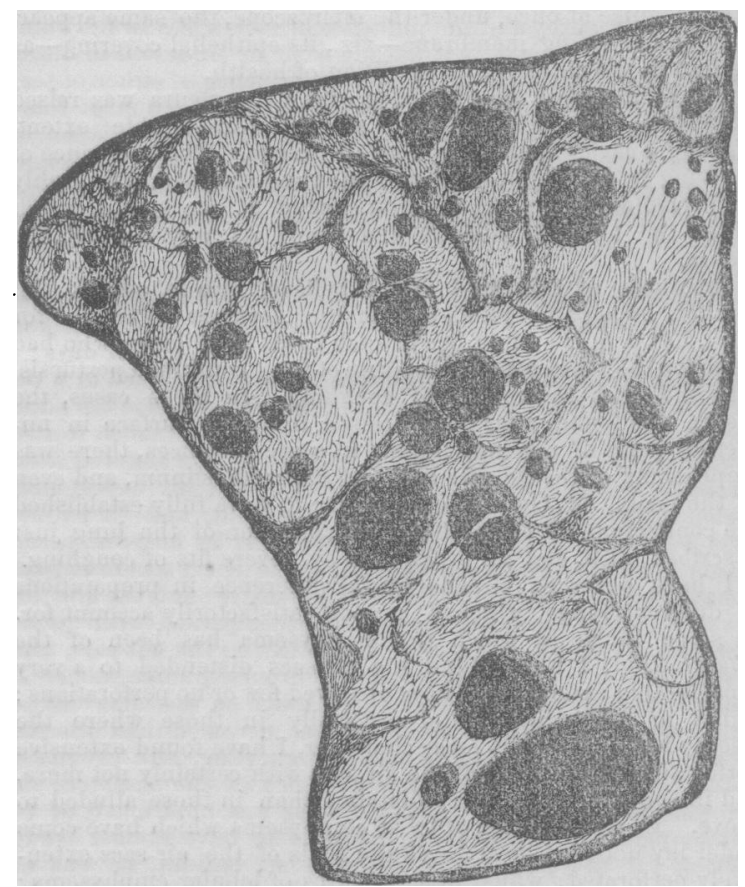

Fig. 10.-Taken from a portion of lung very emphysematous. It shows the appearanee of the air-sacs in a dilated condition, with their partitions much ruptured, and full of large perforations. (Drawn by means of the camera lucida.) The black spots shew the perforations. The carities had a diameter of 1-16th, 1-20th, and 1.27 th of an inch. 
dergo other changes, and are reduced to mere membranous shreds or thin fibrous cords, passing in various directions, traversing, in fact, the distended sacs, two or more of which, by the destruction of their walls, have united to form a single cavity. These cavities occasionally assume a large size, and project from the margin of the lung, forming appendages, and being connected with it merely by a stalk.like process. (See Figs. 10 and 11.)
These appendages differ much in form and volume. In the latter they vary from the size of a nut to that of a pigeon's egg and sometimes they are even larger. As has been observed by Louis, their elongated and rounded form sometimes makes them resemble the swimming bladder of a fish. They will sometimes empty themselves from a single puncture; at others, two or more punctures are required before they will completely collapse. They present in their interior, cavities varying in

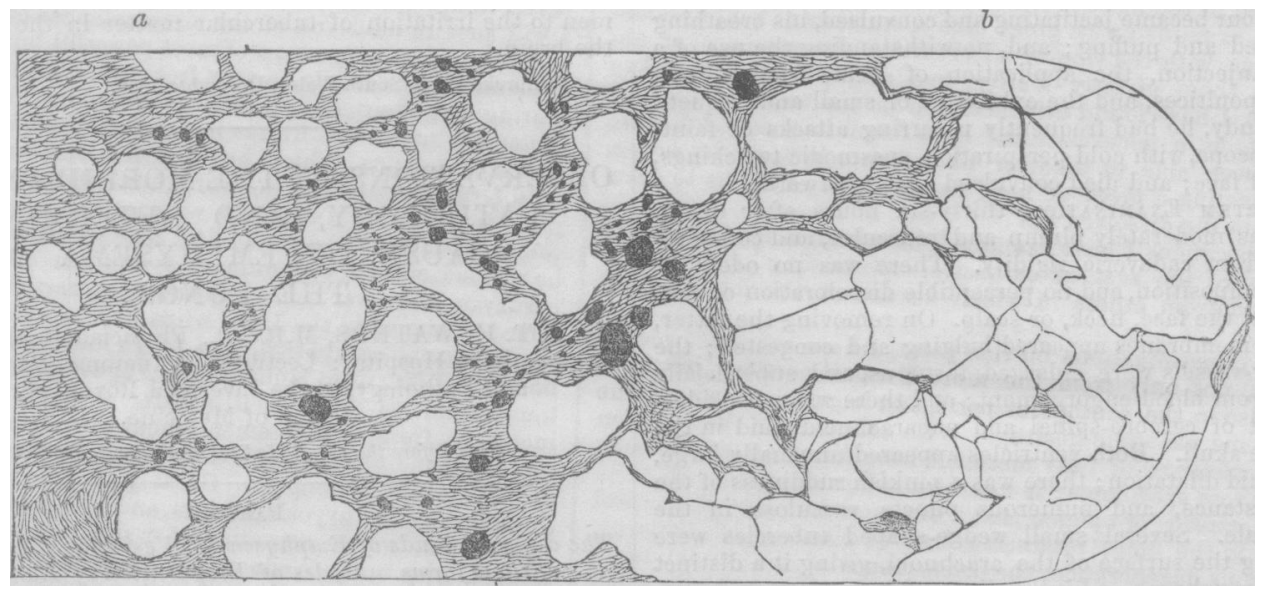

Fig. 11.-Shews the appearance of a piece of highly emphysematous lung, taken from the margin of the lung, where a distinct bulging or "vesicle" existed. The white spaces denote the openings of the divided air.sacs; the black spots, the perforations of their walls; and the lighter parts represent the walls themselves. At $a$, the lung. tissue is tolerably healthy, at $b$, the walls of the air-sacs are broken up, and the membranous shreds and septa llone remain. (Taken by means of the camera lucida, and magnified to one-half the linear dimensions of Figs. J aud 6 .)

size, and traversed at all angles by thin membranous shreds. At the distal extremity of the appendages these cavities are larger, and they diminish towards the end where the appendages join the lung.

If we examine the inner surface of the appendages I have just described, or of a portion of emphysematous lung which has reached an ordinary degree of development, we find abundant evidence that the cavities are formed by the dilated air.sacs. We recognise at once, under the microscope, the same appearance of the lining membrane-viz., its epithelial covering-as we find in the air-sacs in a condition of health.

I have met with one instance where the pleura was raised from the lung-substance, to a very considerable extent, forming a cavity as large as a small orange. The substance of the lung from which the pleura was thus stripped, was highly emphysematous, and an opening existed at one spot, through which the air had escaped, and found its way beneath the pleura.

Instances of this kind are, I believe, rare in adults, $M$ Guillot, in a paper published in the Archives Générales for 1853 , has detailed a number of such cases in children, who had been for the most part the subjects of long continued pertussis, attended with severe fits of coughing. In these cases, the pleura was found, after death, raised from the surface in numerous parts of the lung; and, in some instances, there was emphysema of the cellular tissue of the mediastinum, and even of the neck. M. Guillot seems to me to bave fully established the connection of the pathological condition of the lung just described, with, and its production by, severe fits of coughing.

I have observed an anatomical difference in preparations of different lungs, which I cannot satisfactorily account for. In some lungs in which the emphysema has been of the lobular kind, I have found the air-sacs distended to a very considerable extent, but I have observed few or no perforations ; whilst in other lungs, and especially in those where the disease has been of the lobar character, I have found extensive perforation of the walls of the air-sacs with certainly not more, and in some instances less, dilatation than in those alluded to above. In all the cases of lobar emphysema which have come under my notice, I have found the walls of the air-sacs extensively perforated; whilst in some cases of lobular emphysema ; this condition has not existed. These facts would seem to indicate that, in the cases where rupture takes place under a dilatation, which in others does not produce such rupture, there must be some degeneration which renders the tissue more liable to give way.
If we examine the condition of the blood-vessels in an emphysematous lung, we have evidence of the cause of its anmmic appearance. We find in the earlier stages of the disease when there is simply a dilatation of the lung tissue, that the capillaries of the pulmonary plexus are wider apart than in a state of health; the meshes formed by them are larger. As the walls become perforated, and the sacs further distended and broken, the capillaries become ruptured, and hence we have one cause of the hæmoptysis which occasionally attends the progress of the disease; although this symptom does not necessarily follow from the pathological condition just alluded to.

If we examine a piece of lung in a condition of extreme emphysema we observe a number of small blood-vessels taking their course in the membranous septa and shreds, which $I$ have previously described as traversing the cavities; the vascularity of these parts is exceedingly slight, and but little, or no, respiratory function can be performed by them.

In examining the condition of the bronchial tubes in emphysematous lungs, I have found them dilated, especially in old standing cases. Where bronchitis has existed, the mucous membrane of the larger tubes has been red, injected, and. somewhat thickened; but I have usually observed that the smaller tubes were pale, and exsanguine; although, in some instances $I$ have found them filled with muco-purulent matter. I have not seen ulceration present in any case.

If the smaller bronchial tubes of two portions of the same lung be examined, the one portion being healthy, and the other emphysematous, the tubes of the former will be found of a darker colour than those of the latter. This results in part from the diminished vascularity of the pulmonary tissue in the emphysematous portion, as compared with that of the other; and in part from the same condition of the bronchial mucous membrane.

An alteration of tissue $I$ have frequently observed in old cases of lobar emphysema is increased development of the circular fibres of the bronchial tubes. In the smaller tubes, the fibres become more distinct than in a state of health, and they are more readily recognisable.

A very important question in connection with emphysema, and one which demands the most serious attention of the pathologist is, whether the disease is preceded by, or attended with, any degeneration of tissue; for upon a recognition of the exact nature of the changes which the lung tissue undergoes, and which result in the loss of its healthy elasticity, must be based the means to be resorted to, to arrest the progress of the 
degeneration (if there be any), to restore the lung substance to its healthy state, and thus prevent the further development of a disease, which admits of no cure when once established.

Mr. Rainey, in a specimen he examined, found " the pulmonary membrane studded with fatty matter," and he thought this deposit of fat preceded the formation of the perforations which take place in the disease. The observations of $\mathrm{Mr}$. Rainey on this point have not been confirmed by subse quent inquirers, and the conclusion he arrived at cannot be considered in the light of an established fact.

I have examined with great care a number of emphysematous lungs, with the view of ascertaining the presence or absence in them of fatty matter, and although in one or two instances I have found evidence of it in the walls of the airsacs, yet, as a general rule, I have found it absent. I have neither been able to discover its presence by the microscope, nor by heating a piece of lung tissue on paper, so as to get a stain from the dissolved fat.

The specimens I have examined, have been taken from lungs which were the seat of extensive, old-standing lobar emphy sema, and from others in which disease of the lobular kind existed.

In my investigations on this point, $I$ have examined pieces of lung, not only from the more diseased portions, but from those also in the contiguous parts, and from others where the lung tissue was quite healthy. With reference to the question of the existence of fatty matter, $I$ have found no difference in any of these specimens; it was, as a rule, absent from all.

Dr. Jenner is of opinion that " the most frequent anatomical change in the lung," producing loss of elasticity of its tissue, " is fibrous degeneration, the consequence of the exudation of that variety of lymph which escapes from the capillaries when they are the seat of slight but long continued congestion."

I have examined, microscopically, several specimens of em physematous lungs, with the view of ascertaining whether I could observe any difference in their elastic fibres, as compared with those of healthy lungs. My results are, at present, so imperfect, that I must refrain from giving them; contenting myself with merely expressing an opinion that, in many cases of emphysema, especially of the lobar kind, some degeneration of the pulmonary tissue does exist, into the nature of which, it behoves all pathologists carefully to inquire.

Interlobular Emphysema. This consists of an infiltration of air into the areolar tissue which exists between the various lobules. I have never seen it as an independent affection, but in almost every case where there has been extensive vesicular emphysema, I have found the interlobular kind existing to a greater or less extent. It is often very partial, and seems to have little tendency to spread. At other times (especially in cases of lobar vesicular emphysema) it is quite easy to pass the air from one lobule to another, so that it must traverse extensively the interlobular areolar tissue. From the communica. tion of this tissue with that beneath the pleura, it is easy to see that the latter may become stripped from the lung by air which has first found its way between the lobules; and from its connection with the tissue surrounding the bronchial tubes and blood-vessels, and with that in the mediastinum, we can explain the occurrence of those cases in which emphysema of the cel. lular tissue of the neck has been produced by violent and long continued expiratory efforts.

\section{[To be continued.]}

\section{REMARKS ON OBSTRUCTION OF THE BOWELS: WITH CASES.}

By Edward Copeman, M.D., M.R.C.P., F.R.C.S., Physician to the Norfolk and Norwich Hospital.

[Continued from page 957.$]$

CASE vIr. Fatal Obstipation frou Impacted Gall-Stones. My attendance was requested on September 3rd, 1858, by Mr. - a farmer, 50 years of age, of plethoric habit and considerable bulk and strength. He was accustomed to high living, frequently taking malt liquor and other stimulants, but not to excess. A month ago he was attacked with severe pain in the right side, supposed to be from gall-stones, followed by jaundice. On Monday last, after recovering from his former attack, he was seized with pain in the body, resulting in complete obstruction of the bowels; and when I saw him this morning, he was constantly vomiting dark green matter; he had cold extremities, clammy perspirations, imperceptible pulse, and although perfectly sensible, was fast sinking. $\mathrm{He}$ died soon after I left him, and I was favoured with the following interesting report of his case, by my friend, Mr. - , who regularly attended him.

"Poor H. - died at about one o'clock on Friday; at that time he asked for a draught of harvest beer, which he swallowed eagerly; immediately afterwards, severe vomiting took place, and he expired in the effort. You could bardly have reached the first station when this took place. I obtained leave for a post mortem examination and performed it yesterday, twentyseven hours after death. Before describing the appearances, I will just remind you of the circumstances of the case. He was 50 years of age, a man of most powerful build, and of energetic and active habits, a very free liver, but not intemperate. During the fourteen years I have attended his family, I can remember no serious illness from which he has suffered; an attack of sciatica, which has recurred from time to time, in a mild form, having been the worst, until July 2 nd of the present year, from which (although with rather a long interval of comparative health) we may date his last attack. On that day he was seized with the ordinary symptoms of gall-stones, the paroxysms however being unusually protracted and severe. I attended him until the 15th, when be was well enough to pursue his usual occupation, and to go long journeys, and I saw no more of him until Monday last, August 30th, when the symptoms returned with even greater severity. Vomiting was incessant from the commencement to his death, neither food nor medicine remaining in the stomach for more than an hour or two at the very longest. Great pain and excessive tenderness in the right hypochondrium, and subsequently in the right iliac region, with a sensation of "stoppage somewhere" distressed him until Wednesday, September 1st, when all pain and tenderness ceased, to return no more; and vomiting of dark green fluid, with failing pulse and powers remained. On Friday morning, September 3rd, as you will remember, we found him pulseless, with cold clammy extremities, and, in short, in a state of collapse. The bowels had refused to act in spite of the administration of large soap, turpentine, and castor oil enemata, and free doses of calomel and opium at'first, and afterwards of calomel and colocyuth; none of which, however, were retained a sufficient time to produce effect. The head remained perfectly clear until the last moment.

"Post Mortem Examrnation twenty-seven hours after death. Decomposition was advancing. The face and the upper part of the chest were greatly congested, of a dark purple colour and emphysematous. The body was loaded with fat. There was copious discharge of bloody fluid from the mouth and nares. The lungs were sound. The heart was rather large and flabby. Nothing else worthy of note was found. The abdomen was much distended and hard; a large quantity of offensive gas escaped when the cavity was opened. The stomach and intestines were enormously distended with air. The liver was very small. The stomach was apparently healthy. The duodenum was of a dark chocolate colour, both on its peritoneal and inner surface; its mucous membrane was broken down, and a perforation as large as a florin existed in the position of the mouth of the common bile duct. This tube, together with the entire gall-bladder, were completely gone and disorganised, their place being apparently supplied by condensed and dark connective tissue, which, however, presented no stain of bile, and which had (I suppose recently) become torn, and nearly en. tirely detached from the intestine. The cavity of the duodenum contained a gall-stone of about the size of a musket-bullet, of a cylindrical form, flattened at both extremities by attrition with other concretions, and weighing 84 grains; two more were found in the ileum, weighing respectively $64 \frac{1}{2}$ and 157 grains, the latter, also, being flattened at each end, cylindrical, and larger than a pigeon's egg; a fourth gall-stone, weighing 52 grains, was found loose in the abdomen. The liver was very small, not above half its usual size; its parenchyma was of a pale greenish-yellow hue; the gall-bladder was gone, as before stated. The ileo-colic valve was healthy, as also was the large intestine. The vermiform appendix contained a few pieces of old, hardened fæces, but was neither enlarged nor inflamed. The other organs were apparently healthy. The following, then, were the diseased appearances:-

"Liver. Parenchyma, pale yellowish-green; reduced to half its normal size.

"Gall.bladder and Ducts destroyed; their place being supplied by condensed and dark connective tissue which was torn from the duodenum.

"Duodenum, shewing a large perforation in the position of the entrance of the bile duct; dark chocolate colour without; 